\title{
Dépistage de mammites subcliniques chez des vaches Goudali en lactation au Nord Cameroun
}

\author{
H. Gambo ${ }^{1}$ C. Agnem Etchike ${ }^{2}$
}

\section{Mots-clés}

Bovin - Zébu Goudali - Mammite bovine - Numération cellulaire somatique - Cameroun.

\begin{abstract}
Résumé
L'objectif du présent travail a été d'étudier la prévalence des mammites subcliniques dans les exploitations bovines laitières de la Vina. Le comptage des cellules somatiques par la méthode microscopique directe et le test de Schalm (Cmt, California Mastitis Test) ont été utilisés pour analyser des échantillons de lait prélevés. Les prélèvements de lait de mélange ont été effectués dans 37 exploitations de la région. Un taux moyen de $1240 \pm 765 \times 10^{3}$ cellules par millilitre de lait a été obtenu avec une variation significative liée à la taille du troupeau $(p<0,05)$. Le suivi a été régulier et s'est étalé sur six mois dans trois exploitations différentes. Il a consisté à analyser mensuellement le lait de mélange et les échantillons individuels de lait de vaches en lactation. Les résultats du comptage cellulaire direct du lait de mélange ont montré un taux cellulaire moyen de $834 \pm 204 \times 10^{3}$ cellules par millilitre de lait, soit l'équivalent d'un score $\mathrm{Cmt} 2$ révélateur d'une infection mammaire. Aucune différence mensuelle significative n'a été enregistrée. L'analyse de 504 échantillons individuels a permis de mettre en évidence l'effet significatif du rang de lactation et du stade de lactation sur les résultats du comptage cellulaire du lait. Les résultats ont montré que les femelles zébus Goudali des zones tropicales, bien que faiblement productrices de lait, étaient prédisposées aux mammites subcliniques. Ainsi, le comptage des cellules somatiques a semblé être un outil utile à développer à petite ou à grande échelle pour un dépistage systématique et régulier dans un programme intégré de lutte contre les mammites.
\end{abstract}

\section{INTRODUCTION}

$\mathrm{Au}$ Cameroun et particulièrement dans la zone de Ngaoundéré, l'introduction de l'élevage bovin laitier a été facilitée tant par le projet laitier canadien que par les programmes d'insémination artificielle et de sélection génétique pilotés par l'Institut de recherche agronomique pour le développement (Irad) à Wakwa. La rentabilité de ce type d'élevage dépend de la maîtrise de l'alimentation et du contrôle de certaines pathologies comme les infections mammaires.

La mammite est un état d'inflammation de la glande mammaire résultant de l'action de micro-organismes pathogènes très variés. Ces derniers attaquent et endommagent les tissus sécrétoires qui réagissent très souvent contre l'agression par la mobilisation des leucocytes polynucléaires neutrophiles dans la région de l'infection $(16,19)$. Elle se rencontre généralement chez les vaches en

1. Université de Ngaoundéré, BP 592, Ngaoundéré, Cameroun

Tél. : +2372252598; fax : +2372251694

2. Université de Ngaoundéré, Faculté des Sciences, BP 454, Ngaoundéré, Cameroun lactation et entraîne, d'une part, la baisse de la production de lait et, d'autre part, la baisse de la qualité hygiénique et nutritive du lait et de ses produits dérivés $(8,9,12,20)$.

Cette affection, très répandue dans le monde et particulièrement dans les exploitations laitières des pays du Nord, tend à être négligée chez les races tropicales faiblement productives (18). La vache Goudali, dont la production moyenne est de $500 \mathrm{~kg}$ de lait par lactation (14), présente rarement des formes cliniques de mammites (4). Les résultats d'une enquête réalisée auprès des services d'élevage de Ngaoundéré ainsi que les rapports d'activité de la délégation montrent que, sur un total annuel de 12325 bovins présentés en consultation en 1992, seulement 14 cas de mammites cliniques ont été observés (4). Cela n'exclut cependant pas l'existence de formes subcliniques, sans symptômes apparents ni altération visible du lait. Dans ces formes, seule l'augmentation du taux de globules blancs et de cellules épithéliales du lait est décelée par divers tests de comptage cellulaire $(1,5,8)$. Ainsi, le comptage des cellules somatiques du lait est devenu un élément d'appréciation de l'état sanitaire global de la mamelle, d'estimation de la prévalence des mammites dans le troupeau et d'évaluation de la qualité du lait destiné à la consommation $(5,22)$. La présente étude a eu 
pour objectif, d'une part, d'évaluer la prévalence des mammites subcliniques dans les exploitations à vocation laitière afin d'envisager des solutions d'amélioration et, d'autre part, de mettre sur pied des méthodes faciles et économiques de dépistage précoce des mammites pour les éleveurs des zones tropicales.

\section{MATERIEL ET METHODES}

\section{Zone de l'étude}

L'étude a été conduite dans l'Adamaoua et, plus précisément, dans la zone de Ngaoundéré et de ses environs. C'est une région de transition entre le Sud forestier et les savanes soudaniennes du Nord. Elle se situe entre $6^{\circ} 20^{\prime}$ et $7^{\circ} 40^{\prime}$ de latitude nord et 11 et $15^{\circ}$ de longitude est. Son altitude varie entre 900 et $1500 \mathrm{~m}$. Les précipitations monomodales couvrent généralement sept mois, avec une moyenne de $1500 \mathrm{~mm}$ pour 120 à 150 jours de pluies par an. Les températures moyennes oscillent entre 23 et $25^{\circ} \mathrm{C}$ (15). Toutes ces données confèrent à cette zone un caractère propice au développement de l'élevage. Le cheptel bovin, constitué à majorité d'animaux de race Goudali, a été estimé à 1800000 têtes en 1994, soit 28 p. 100 du cheptel bovin national (15).

\section{Exploitations}

La facilité d'accès des exploitations et la disponibilité des éleveurs ont été les seuls critères de choix. L'étude s'est déroulée à deux niveaux :

- un suivi mensuel régulier de trois exploitations à vocation laitière dans les régions de Bini, Mballang et Maoui ;

- une enquête transversale sur des exploitations bovines non spécialisées en production de lait. L'enquête a été menée entre les mois de mai et de novembre qui correspondent à la saison des pluies, période pendant laquelle la disponibilité en fourrage permet une production laitière optimale.

Des trois exploitations ayant fait l'objet du suivi mensuel, les exploitations E2 et E3, quasi identiques d'après les critères retenus (tableau I), ont été différentes de E1.

Pendant la période de tarissement dont la durée moyenne varie de 3 à 5 mois dans la région (14), seules les vaches de l'exploitation E1 ont été systématiquement soumises à une antibiothérapie générale à base d'oxytétracycline 20 p. 100 longue action et à une injection intra-mammaire de benzathine cloxacilline après la dernière traite.

L'enquête transversale, dont l'objectif a été d'estimer le niveau moyen de contamination dans la zone, a été menée dans 37 exploitations différentes. C'étaient en général des élevages de type extensif pour lesquels la production laitière n'était pas une source de revenus, le lait trait étant destiné à la consommation familiale.

Le niveau d'hygiène a été médiocre dans l'ensemble. Avant la traite, même le simple lavage des trayons a été négligé. Il n'y a pas eu de trempage des trayons ni d'utilisation de serviettes individuelles. La traite a été manuelle et s'est passée à l'étable ; le traitement anti-infectieux au tarissement n'a pas été appliqué. Ces exploitations ont été dans leur quasi-totalité similaires aux exploitations E2 et E3 ayant fait l'objet du suivi.

En fonction de l'effectif, ces exploitations ont été subdivisées en trois classes distinctes :

- Cl-1, 15 à 20 vaches laitières $(\mathrm{n}=12)$;

- $\mathrm{Cl}-2,21$ à 40 vaches laitières $(\mathrm{n}=17)$;

- Cl-3, plus de 40 vaches laitières $(n=8)$.

\section{Prélèvements}

Deux types de prélèvements ont été effectués : un prélèvement de lait individuel issu des quatre quartiers de la mamelle de chaque vache en lactation et un prélèvement de lait de mélange (lait issu de la traite de toutes les vaches en lactation présentes dans l'exploitation). Le premier type de prélèvement, effectué uniquement dans les exploitations suivies mensuellement, a permis d'apprécier le niveau individuel d'infection mammaire. Le deuxième type, utilisé pour évaluer le niveau de contamination moyen du cheptel, a été effectué dans toutes les exploitations visitées. Le lait des quartiers individuels a été prélevé directement après nettoyage de la mamelle et expulsion des premiers jets de lait. Tous ces prélèvements (flacons de $25 \mathrm{ml}$ ) ont été identifiés et acheminés au laboratoire dans des conditions strictes de réfrigération $\left(4^{\circ} \mathrm{C}\right)$.

\section{Tests}

Deux tests ont été appliqués sur chaque échantillon prélevé.

1. Le test de Schalm (California Mastitis Test ou Cmt). Son principe repose sur l'utilisation d'un corps tensioactif (le teepol à 10 p. 100) qui provoque l'éclatement des cellules et la précipitation de leur Adn et d'une solution de pourpre de bromocrésol à $1 / 10000^{\mathrm{e}}$ qui joue le rôle d'indicateur de $\mathrm{pH}(2,3,9)$. Deux millilitres de lait à tester sont mélangés à une même quantité de réactif (Leucocytest) à température ambiante. La réaction a été numérotée de 0 à 4 en fonction du niveau d'infection $(1,6,24)$ et la notation $\mathrm{Cmt1}$ a correspondu à un résultat douteux (tableau II) (6).

2. Le comptage des cellules somatiques (Ccs) par la méthode de Breed et Prescott. Les cellules du lait sont comptées directement au microscope après coloration des noyaux. Pour réaliser ce comptage, $0,01 \mathrm{ml}$ de lait a été étalé sur une surface de $1 \mathrm{~cm}^{2}$ d'une lame microscopique ordinaire. Le colorant de Broadhurstpaley a été utilisé pour la préparation des films. La lecture a été faite sur 25 champs microscopiques non surperposés, choisis au hasard. Le facteur multiplicatif utilisé a été celui de Breed et Prescott dont la valeur est de $20000(17,21)$.

Tous les échantillons prélevés ont été soumis dans un premier temps au Cmt dans l'exploitation même. Le second test, exigeant plus de temps et de matériel a été réalisé au laboratoire.

\section{Tableau I}

Caractéristiques des exploitations

\begin{tabular}{|c|c|c|c|c|c|}
\hline Exploitations & Effectif & $\begin{array}{l}\text { Système } \\
\text { d'élevage }\end{array}$ & $\begin{array}{l}\text { Système } \\
\text { de traite }\end{array}$ & $\begin{array}{c}\text { Traitement } \\
\text { de tarissement }\end{array}$ & $\begin{array}{l}\text { Hygiène } \\
\text { de la traite }\end{array}$ \\
\hline E1 (Maoui) & 24 & Semi-intensif & Manuel au pâturage & + & Lavage des trayons \\
\hline E2 (Bini) & 32 & Extensif & Manuel au pâturage & - & Aucune \\
\hline E3 (Mballang) & 28 & Extensif & Manuel au pâturage & - & Aucune \\
\hline
\end{tabular}




\section{RESULTATS}

\section{Valeur du comptage des cellules somatiques}

Dans le cadre de l'enquête transversale, 37 échantillons de lait de mélange issus de 37 exploitations différentes ont été analysés. La concentration cellulaire moyenne enregistrée a été de $1240 \pm 765$ x $10^{3}$ cellules par millilitre de lait.

Une forte variation a été notée entre les exploitations. Dans quatre exploitations, les valeurs ont été inférieures à 400 x $10^{3}$ cellules/ml. Dans sept exploitations, elles ont été comprises entre 400 et $800 \times 10^{3}$ cellules/ml. Dans les 26 restantes, elles ont été supérieures à $800 \times 10^{3}$ cellules $/ \mathrm{ml}$ de lait. Le taux le plus élevé a été de $2830 \times 10^{3}$ cellules $/ \mathrm{ml}$ alors que le taux le plus bas a été de $260 \times 10^{3}$ cellules $/ \mathrm{ml}$. Ces exploitations ayant toutes été de type extensif, sans hygiène de la traite, la taille du troupeau a été le seul facteur pris en considération dans les analyses.

\section{Effet de la taille du troupeau}

Les taux cellulaires les plus élevés ont été enregistrés dans les grands troupeaux (classe 3) alors que les taux les plus bas l'ont été dans les petits troupeaux (classe 1) (tableau III). L'analyse de variance a montré une différence significative entre ces deux classes $(\mathrm{P}<0,05)$. Il est à noter que les valeurs moyennes enregistrées dans les différentes classes ayant toutes été supérieures à $1000 \times 10^{3}$ cellules $/ \mathrm{ml}$, tous les types ont été contaminés mais à des degrés divers.

\section{Lait de mélange}

Le lait de mélange de chacune des trois exploitations a été mensuellement analysé. Les résultats enregistrés ont montré une variation par exploitation et par mois de prélèvement (tableau IV).

Aucune différence significative n'a été relevée entre les mois de prélèvement. Les résultats moyens des exploitations E2 et E3,

\section{Tableau II}

Lecture et interprétation du test de Schalm

\begin{tabular}{lccc} 
Aspect & Réaction & Notation & Concentration cellulaire \\
\hline $\begin{array}{l}\text { Mélange fluide } \\
\text { Floculat très fin qui disparaît après agitation }\end{array}$ & Négative & 0 & 0 à 200000 \\
& $\begin{array}{c}\text { Douteuse }+1 \\
\text { (état de trace) } \\
\text { Positive }+2\end{array}$ & 1 & 150000 à 500000 \\
$\begin{array}{l}\text { Floculat très net sans tendance à la gélification } \\
\text { par endroits, consistance du blanc d'œuf }\end{array}$ & Positive +3 & 3 & 400000 à 1500000 \\
Gel épais à la consistance du crachat & Positive +4 & 4 & $>500000$ à 5000000 \\
\end{tabular}

D'après Oaki, 1990 ; Dumont et coll., 1999 ; Selze, 1999

\section{Tableau III}

Moyenne cellulaire par classe d'effectifs

\begin{tabular}{lccc} 
Classe & Cl-1 & Cl-2 & Cl-3 \\
\hline $\begin{array}{l}\text { Nombre d'exploitations } \\
\text { Moyenne (cellules/ml) }\end{array}$ & 12 & 17 & 8 \\
& $1022 \pm 660 \times 10^{3 a}$ & $1678 \pm 738 \times 10^{3}$ & $2168 \pm 480 \times 10^{3 b}$
\end{tabular}

a vs $\mathrm{b}, \mathrm{P}<0,05$

\section{Tableau IV}

Concentration cellulaire moyenne en $10^{3}$ cellules/ml par exploitation et par mois

\begin{tabular}{lclllrrrrr} 
Exploitation & Effectif & Juin & Juillet & Août & Septembre & Octobre & Novembre & Moyenne/exploitation \\
\hline E1 & 24 & $420(1)^{*}$ & $312(1)$ & $648(2)$ & $532(2)$ & $488(1)$ & $490(2)$ & $482^{\mathrm{a}}$ \\
E2 & 32 & $944(3)$ & $1032(3)$ & $1068(3)$ & $984(2)$ & $1206(3)$ & $1124(3)$ & $1060^{b}$ \\
E3 & 28 & $836(3)$ & $860(3)$ & $904(3)$ & $1048(3)$ & $988(2)$ & $1136(3)$ & $962^{b}$ \\
Moyenne & - & 733 & 734 & 873 & 854 & 894 & 916 & 834 \\
mensuelle & & & & & & & &
\end{tabular}

* Score Cmt

a vs $\mathrm{b}, \mathrm{P}<0,05$ 
statistiquement identiques, ont différé significativement de ceux d'E1 $(\mathrm{p}<0,05)$.

La correspondance entre les scores Cmt mensuels et les concentrations cellulaires mensuelles a été en général conforme aux normes théoriques (tableau IV). Cependant, certains résultats ont montré des divergences.

Dans l'exploitation E1, les concentrations cellulaires de 488 et 490 x $10^{3}$ cellules $/ \mathrm{ml}$ enregistrées respectivement en octobre et en novembre ont été peu différentes, contrairement aux scores $\mathrm{Cmt}$ correspondants (respectivement 1 et 2), indiquant les valeurs limites entre les deux notations. Dans l'exploitation E2, le score Cmt a été plus élevé en juin qu'en septembre alors que la concentration cellulaire du mois de septembre a été légèrement plus élevée que celle de juin. Les résultats enregistrés dans l'exploitation E3 pendant les mois de juin et d'octobre ont été similaires.

\section{Prélèvements individuels}

Pendant les six mois de l'étude, 84 vaches issues des trois exploitations suivies ont été prélevées individuellement chaque mois et 504 échantillons de lait de mélange de quartiers individuels ont été analysés par les deux méthodes (Cmt et comptage microscopique direct). Aucune des vaches n'a manifesté de symptôme clinique de mammite avant le prélèvement. Une analyse statistique a été menée pour apprécier l'effet du rang de lactation et du stade de lactation.

\section{Effet du rang de lactation}

Dans les trois exploitations du suivi, les femelles en production ont été classées selon leur rang de lactation en quatre catégories (tableau V). Les tests $\mathrm{Cmt}$ ont donné des scores de 0 et de 1 le plus souvent chez les primipares et les vaches de $2^{\mathrm{e}}$ et de $3^{\mathrm{e}}$ rang de lactation. Les scores 2 et 3 ont surtout été enregistrés chez les femelles de $4^{\mathrm{e}}$, de $5^{\mathrm{e}}$ rang de lactation et plus. Les scores 4 sont restés rares dans la zone.

Le comptage microscopique direct a donné des résultats peu différents du Cmt (tableau V). La réponse cellulaire a semblé d'autant plus importante que les vaches ont eu un rang de lactation plus élevé. Une différence significative a été notée entre les vaches de $1^{\mathrm{er}}$ rang de lactation et celles de $4^{\mathrm{e}}$, de $5^{\mathrm{e}}$ rang de lactation ou audelà $(\mathrm{p}<0,01)$.

\section{Effet du stade de lactation}

La figure 1 montre l'effet du stade de lactation sur les résultats du comptage cellulaire individuel réalisé dans les trois exploitations. x $10^{3}, 825 \times 10^{3}$ et $1074 \times 10^{3}$ cellules $/ \mathrm{ml}$ ont été enregistrées au $1^{\mathrm{er}}$ mois de lactation, entre les $2^{\mathrm{e}}$ et $4^{\mathrm{e}}$ mois et au-delà du $5^{\mathrm{e}}$ mois.
Le début ( $1^{\mathrm{er}}$ mois) et la fin (au-delà du $5^{\mathrm{e}}$ mois) de la lactation se sont caractérisés par un niveau de comptage élevé qui les a distingués significativement $(\mathrm{p}<0,01)$ de la mi-lactation $\left(2^{\mathrm{e}}-4^{\mathrm{e}}\right.$ mois $)$.

\section{DISCUSSION}

Le taux cellulaire moyen de $1240 \times 10^{3}$ cellules/ml de lait de mélange enregistré dans les exploitations de Ngaoundéré et de ses environs a été un indicateur de la forte proportion de quartiers infectés dans les troupeaux de la région. Il ressort des relations d'équivalences établies par certains auteurs $(7,20)$ qu'une concentration de $1000 \times 10^{3}$ cellules $/ \mathrm{ml}$ de lait de mélange équivaut à une moyenne de 32 p. 100 de quartiers infectés dans le troupeau et une perte estimée à 17,7 p. 100 de la production totale de lait. La concentration enregistrée dans cette étude (1 $240 \times 10^{3}$ cellules $\left./ \mathrm{ml}\right)$ a été équivalente à un taux estimatif de plus de 32 p. 100 de quartiers atteints dans les troupeaux visités, témoignant d'une prévalence élevée des mammites subcliniques dans la zone.

L'effet de la taille du troupeau mis en évidence par l'analyse de variance pouvait s'expliquer par la facilité d'application des mesures de prévention sur un effectif réduit. Plus le cheptel est grand, moins le suivi individuel est assuré et plus le nombre de quartiers atteints est élevé (9). Les pertes économiques liées à un taux cellulaire élevé ne sont pas à négliger dans cet environnement où le revenu du paysan est très bas. Les résultats des études menées par Lescourret et Coulon montrent que les baisses de production laitière observées dans les cas de mammites sont généralement à court terme (13). Ces pertes sont faibles à l'échelle individuelle et restent tributaires de certains facteurs tels que le stade de lactation (13). Cependant, à l'échelle du troupeau, le manque à gagner n'est pas à négliger.

La présence de ces infections mammaires pourrait être attribuée aux mauvaises conditions d'hygiène de traite qui favorise la trans-

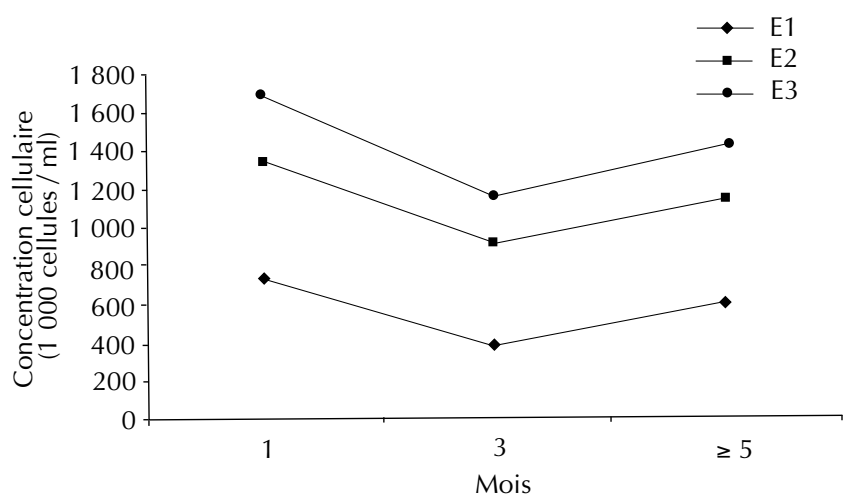

Figure 1 : effet du stade de lactation sur la concentration cellulaire.

Tableau V

Taux cellulaire en $10^{3} / \mathrm{ml}$ par exploitation et par rang de lactation

\begin{tabular}{lccrc} 
Rang de lactation & $\mathbf{1}^{\text {re }}$ lactation & $\mathbf{2}^{\mathbf{e}} \mathbf{- 3 ^ { \mathbf { e } }}$ lactation & $\mathbf{4}^{\mathbf{e}} \mathbf{- 5}$ lactation & $\mathbf{5}^{\mathbf{e}}$ lactation \\
\hline E1 & 250 & 368 & 642 & 665 \\
E2 & 486 & 874 & 1305 & 1576 \\
E3 & 402 & 730 & 1062 & 1142 \\
Moyenne & $380^{\mathrm{a}}$ & $657^{\mathrm{a}}$ & $1003^{\mathrm{b}}$ & $1127^{\mathrm{b}}$
\end{tabular}


mission de l'infection d'un quartier à un autre ou d'une vache à une autre $(9,16,23)$.

Les concentrations cellulaires du lait de mélange enregistrées dans les trois exploitations suivies ont montré dans l'ensemble des chiffres supérieurs à $400 \times 10^{3}$ cellules $/ \mathrm{ml}$, norme hygiénique limite fixée par l'Union européenne $(1,9,22)$. Seule l'exploitation E1 a montré un résultat inférieur à cette norme pendant le mois de juillet. Ces résultats obtenus dans les exploitations phares de la région ont donc été positifs et confirment les résultats de l'enquête transversale. L'exploitation E1 s'est distinguée des autres par son niveau de contamination assez bas. Cette différence pouvait s'expliquer par :

- le caractère semi-intensif du système d'élevage (bon affouragement et complémentation alimentaire, assurant un niveau immunitaire élevé) ;

- les conditions de traite plus satisfaisantes avec un lavage des trayons avant la traite ;

- le traitement systématique au tarissement.

Les taux cellulaires les plus élevés enregistrés chez les vaches de $4^{\mathrm{e}}$, de $5^{\mathrm{e}}$ rang de lactation et au-delà auraient été dus à la baisse des défenses naturelles au niveau de la glande mammaire des vaches âgées. Le canal du trayon devient plus dilaté après chaque lactation, prédisposant davantage la vache aux infections mammaires (1).

Certains auteurs attribuent le taux cellulaire élevé du début de lactation à la perte excessive des cellules épithéliales dans un volume réduit de lait suite à la reprise de l'activité de la glande mammaire après une période de repos $(1,8,21)$. De nombreuses études ont montré que le lait de première semaine de lactation se caractérise par une forte concentration en cellules somatiques, suivie d'une baisse rapide et progressive entre les $25^{\mathrm{e}}$ et $45^{\mathrm{e}}$ jours après le vêlage, pour se stabiliser à un taux bas durant des semaines avant de croître progressivement jusqu'à la fin de la lactation $(10,11,16$, 21). L'élévation du taux cellulaire de fin de lactation aurait simplement été due à l'augmentation de la concentration cellulaire dans un faible volume de lait suite à la baisse physiologique de fin de lactation $(8,16)$. Le colostrum, le lait de fin de lactation et le lait de vaches âgées peuvent donner des réactions faussement positives.

Le Cmt et le comptage direct des cellules au microscope ont donné des résultats sensiblement identiques. L'intervalle de variation cellulaire assez grand pour chaque score $\mathrm{Cmt}$ ne permet malheureusement pas de bien apprécier l'évolution des concentrations cellulaires dans le temps. Les scores Cmt faibles correspondant à de fortes concentrations cellulaires auraient été dus à la présence des cellules dont les noyaux résistent à l'action du réactif (9). Les scores $\mathrm{Cmt} 3$ enregistrés en juin dans les exploitations E2 et E3 sont apparus comme des réactions assez exagérées. Elles auraient pu être dues à l'action du réactif sur les cellules désintégrées $(5,9)$. Dans la pratique, le Cmt reste de loin mieux adapté en milieu paysan, car il est plus rapide et il est nécessite peu de matériel et de connaissances techniques. Mais il reste moins précis que le comptage direct des cellules, en particulier pour apprécier les variations de faibles amplitudes.

\section{CONCLUSION}

Les résultats du présent travail ont montré une concentration cellulaire moyenne de $1240 \times 10^{3}$ cellules/ml de lait de mélange dans les exploitations de la région de Ngaoundéré. Ce taux est un indicateur d'une prévalence élevée de mammite subclinique dont l'impact sur la production quantitative et qualitative du lait n'est pas à négliger. Les résultats relevés au niveau des trois élevages suivis régulièrement semblent indiquer que les mauvaises condi- tions d'hygiène de traite et la mauvaise conduite du troupeau ont constitué les probables facteurs de risque. Il apparaît important d'effectuer un diagnostic précoce par le comptage des cellules du lait pour contrôler cette forme subclinique. Cependant, le rang de lactation et le stade de lactation sont des facteurs qui interagissent sur cette numérotation cellulaire. Le $\mathrm{Cmt}$, bien que très pratique et rapide, est malheureusement subjectif, moins précis que le comptage direct et difficile à utiliser pour apprécier les variations cellulaires dans le temps. Les deux tests sont néanmoins complémentaires dans le dépistage systématique précoce des mammites subcliniques.

Au terme de cette étude les auteurs recommandent :

- de vulgariser l'application de mesures d'hygiène adéquates, particulièrement lors de la traite (au moins le lavage des trayons) ;

- de traiter systématique les mammites cliniques en respectant les règles de base (traitement antibiotique précoce, massif et soutenu effectué après des traites complètes, nettoyage et désinfection du trayon des quartiers à traiter, et suivi d'un délai d'attente avant d'utiliser le lait pour la consommation humaine) ;

- de vulgariser l'utilisation régulière $\mathrm{du} \mathrm{Cmt}$ au niveau de chaque exploitation sur les quartiers de toutes les vaches en lactation tous les trois mois (ou, mieux, tous les mois) et dans tous les centres de collecte de lait ;

- dans la mesure du possible, de faire en sorte que le test soit effectué par la même personne qui notera de la même manière pour limiter la subjectivité du test ;

- de réformer les vaches aux mammites non guéries, à mammites récidivantes ou à quartier fibrosé ;

- d'entreprendre une étude sur de nombreux cas, en station et hors station, pour préciser les limites des notations obtenues sur les niveaux de cellules somatiques dans les conditions locales, particulièrement la limite inférieure de la notation $\mathrm{Cmt} 2$ qui peut être considérée comme positive.

\section{BIBLIOGRAPHIE}

1. OAKI I., 1990. Diurnal variation in count and composition of somatic cell in milk and characteristics related infection mastitis. In: Int. Symp. Bovine Mastitis, National Mastitis Council, Indianapolis, IN, USA, 13-16 september 1990, p. 412-418.

2. BENNET G., 1993. What to expert from sire selection to lower somatic cell count. In: 32nd Annual Meeting National Mastitis Council, Kansas City, KS, USA, 4-7 October 1993, p. 65-72.

3. BOSSE P., 1982. Base d'un plan de prévention des mammites bovines et difficulté de mise en place. Thèse Doct. vét., Faculté Médecine Créteil, France, 65 p.

4. DAWA O., 1992. Rapport annuel. Ngaoundéré, Cameroun, ministère de l'Elevage, des pêches et industries, $76 \mathrm{p}$.

5. DOHOO J.R., LESILE K.E., 1990. Evaluating of changes in somatic cell count as indicator of new intramammary infection. In: Int. Symp. Bovine Mastitis, National Mastitis Council, Indianapolis, IN, USA, 13-16 september 1990, p. 320-325.

6. DUMONT P., PONSART C., HUMBLOT P., GUERIN B., 1999. Etude de la réaction de Schalm au cours du contrôle de la fonction sexuelle chez le jeune taureau Normand. Elev. Insémin., 292 : 3-16.

7. EBERHART R.J., HARMON R., 1987. Current concepts of bovine mastitis. In: Int. Symp. Bovine Mastitis, National Mastitis Council, 3rd Edn, Arlington, VA, USA, $47 \mathrm{p}$.

8. ELVINGER F., NATZKE R.P., 1992. Elements of mastitis control. Large dairy herd management. Am. Dairy Sci. Assoc., 440-447.

9. FADRIG A., 1988. Contribution à l'étude d'un programme antimammite dans six élevages laitiers de Sodea. Thèse Doct. vét., lav, Rabat, Maroc, $137 \mathrm{p}$.

10. HARMON R.J., RENEAU J.K., 1993. Factors affecting somatic cell count in milk. In: 32nd Annual Meeting National Mastitis Council, Kansas City, KS, USA, 4-7 October 1993, p. 243-289. 
11. KENNEDY B.W., SETHAR M., TONGA A., MOXLEY J., 1982. Environmental factors influencing test day somatic cell counts in Holsteins. J. Dairy Sci., 65: 275-280.

12. KIRK J.H., 1984. Programmable calculator program for linear somatic cell counts scores to estimate mastitis field losses. J. Dairy Sci., 67: 441-444.

13. LESCOURRET T., COULON J.B., 1994. Modeling the impact of mastitis on milk production by dairy cows. J. Dairy Sci., 77: 2289-2301.

14. MBAH D.A., MBANYA J., MESSINE O., 1998. Performance of Holsteins, Jerseys and their zebu crosses in Cameroon: preliminary results. Sci. Technol. Rev., Cameroon, 3: 115-126.

15. MBAHE R.E., 1998. Résultats de recherche agricole pour le développement en zone agro-écologique de hautes savanes guinéennes (Adamaoua). In : Comité régional des programmes, 27-28 octobre 1998. Ngaoundéré, Cameroun, Irad, 17 p.

16. MILLET V., 1988. Mammites : Attention danger ! Revue fr. Génét Reprod., 50 : 42-44.

17. PACKARD V.S., GINN R.E., 1985. Methods to detect abnormal milk. In: Van Horn Ed., Standard methods for the examination of dairy products, 15th edn. Wilcox, p. 219-237.

\section{Summary}

Gambo H., Agnem Etchike C. Detection of Subclinical Mastitis in Lactating Gudali Zebus in North Cameroon

The objective of the study was to assess the prevalence of subclinical mastitis in bovine dairy farms of Vina. Milk samples were analyzed by the direct somatic cell count (SCC) method and the California Mastitis Test (CMT). Bulk milk was sampled in 37 dairy farms. Mean SCC was $1240 \pm 765 \times 10^{3}$ cells per milliliter of milk and varied significantly with the herd size $(p<0,05)$. Monitoring was regular and covered a six-month period in three separate farms: bulk milk and individual milk samples from lactating zebu cattle were monthly analyzed. Mean direct SCC was $834 \pm 204 \times 10^{3}$ cells per milliliter of bulk milk, equivalent to a CMT 2 score (sign of mastitis). Monthly differences were not significant. Results from 504 individual milk samples showed that parity and the stage of lactation significantly affected SCC. Although Gudali zebu females of tropical areas yield lesser milk, the results showed that they were still prone to subclinical mastitis. Therefore, SCC seemed to be a useful tool to be developed on a small or larger scale within an integrated control program so as to detect systematically and regularly mastitis.

Key words: Cattle - Gudali zebu - Bovine mastitis - Somatic cell count - Cameroon.
18. REGE J.E., ABOAGYE G.S., AKAH S., AHUNU B.K., 1994. Crossbreeding Jersey with Ghana Sokoto Gudali cattle in a tropical environment: Additive and heterotic effects for milk production, reproduction and calf growth traits. Anim. Prod., 59: 21-29.

19. RENEAU J.K., 1986. Dairy herds performance evaluation: Mastitis monitors. In: Proc. Int. Symp. Bovine Mastitis, National Mastitis Council, 18, p. 38-49.

20. RENEAU J.K., 1990. Monitoring mastitis milk quality and economic losses in herds. In: Int. Symp. Bovine Mastitis, National Mastitis Council, Indianapolis, IN, USA, 13-16 september 1990, p. 326-333.

21. SCHALM O.W., CARROL E.J., JAIN N.C., 1971. Bovine mastitis. Philadelphia, PA, USA, Lea and Febriger, p. 94- 157.

22. SELZE J.C., 1999. Taux cellulaires élevés, qui est responsable ? Mammites cliniques ou subcliniques? Bull. tech. Insémin. artif., $91: 31-33$.

23. SPENCER S.B., 1992. Milking machine function and cleaning. Large dairy herd management. Am. Dairy Sci. Assoc., 475-486.

24. SPENCER S.B., PANKEY J.W., 1990. How to use somatic cell count information. Hoard's dairyman. Natl Dairy Mag., 59.

Reçu le 08.09.1999, accepté le 17.10.2001

\section{Resumen}

Gambo H., Agnem Etchike C. Sondeo de las mastitis sub clínicas en las vacas en lactación en el norte de Camerún

El objetivo del presente trabajo fue el de estudiar la prevalencia de las mastitis sub clínicas en los establecimientos bovinos lecheros de Vina. Con el fin de analizar las muestras de leche, se realizó un conteo de células somáticas, mediante el método microscópico directo y el test de Schalm (Cmt, California mastitis test). Las muestras de leche provenientes de mezclas se obtuvieron en 37 establecimientos de la región. Se obtuvo una tasa media de $1240 \pm 765 \times 10^{3}$ células por mililitro de leche, con una variación significativa, ligada al tamaño del hato $(p<0,05)$. El seguimiento fue regular, con una duración de seis meses, en tres explotaciones diferentes. Consistió en el análisis mensual de la leche de mezclas y de las muestras individuales de leche de vacas en lactación. Los resultados del conteo celular directo de la leche de mezclas mostró una tasa celular media de $834 \pm 204 \times 10^{3}$ células por mililitro de leche, o sea el equivalente de un valor de $\mathrm{Cmt} 2$ revelador de una infección mamaria. No se registró ninguna diferencia mensual significativa. El análisis de 504 muestras individuales permitió demostrar el efecto significativo del rango de lactación y del estadio de lactación sobre los resultados del conteo celular de la leche. Los resultados muestran que las hembras cebú Gudali de las zonas tropicales, aunque bajas productores de leche, estaban predispuestas a las mastitis sub clínicas. Por lo tanto, el conteo de las células somáticas parece ser un instrumento útil a desarrollar, a pequeña o gran escala, para el seguimiento sistemático y regular dentro de un programa integrado de lucha contra las mastitis.

Palabras clave: Ganado bovino - Cebú Gudali - Mastitis bovina - Conteo de celulas somaticas - Camerún. 